\title{
Organización espacial del corredor turístico Veracruz-Boca del Río
}

DOI: 10.22403/UQROOMX/TYP05/13

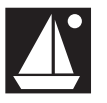

Resumen

Oswaldo Gallegos Jiménez*

Este trabajo muestra un panorama de la organización espacial del corredor turísticoVeracruz-Boca del Río, México. El estudio se divide en tres apartados: en el primero se sintetizan los hechos históricos significativos en el crecimiento del área metropolitana Veracruz-Boca del Río (CTVBR) y de su turismo; en el segundo se especifica el actual espacio turístico del área metropolitana,su estructura interna, así como los recursos y equipamiento turísticos existentes; $y$ en el tercero se expone la dinámica espacial del corredor turístico en estudio.

Palabras | Corredor turístico Veracruz-Boca del Río, estructura urbana, orga-

CLAVE nización espacial, turismo.

*Universidad Nacional Autónoma de Mëxico, Instituto de Geografía / ogj_turismo@yahoo.com.mx 


\section{Introducción}

En México, los espacios turísticos se incrustan a modo de enclaves estructuralmente favorecidos, lo que de manera visible los distingue del resto de la localidad donde se ubican. Si bien tal característica ha sido puesta al descubierto en diversos estudios, sobre todo urbanos, pocas veces se han manifestado las diferencias estructurales y funcionales que los espacios turísticos llegan a tener en su interior. La presente investigación se inserta en la vertiente teórica de la geografía del turismo, cuya finalidad es analizar desde una perspectiva territorial las actividades turísticas; en lo particular, se trata de un estudio de turismo urbano-costero de una de las principales ciudades de México, cuyo objetivo es mostrar la organización espacial del corredor turístico Veracruz-Boca del Río (CTVBR) mediante el examen de su estructura urbana.

Con alrededor de 6500 hectáreas y 545000 habitantes, el área metropolitana Veracruz-Boca del Río (AMVBR) es la más grande del estado de Veracruz y el sitio turístico costero más visitado en el litoral del Golfo de México. Su estructura urbana y vocación turística son resultado de la conjunción, en tiempo y espacio, de una diversidad de características físicas, sociales y económicas locales y nacionales que actualmente evidencia una compleja configuración de su espacio turístico y nuevas formas de ocupación de éste.

\section{Estructura urbana y turismo del AMVBR: evolución y territorio}

El área metropolitana Veracruz-Boca del Río se localiza en la parte este del municipio de Veracruz y en la norte y centro de Boca del Río, en la región Sotavento del estado' (figura I). Su estructura urbana ${ }^{2}$ es producto de una serie de factores físicos y humanos que condicionaron el rumbo primario de su crecimiento: en el caso de Veracruz, fue el entorno pantanoso de su costado norte, la posterior

'http://www.inegi.gob.mx

${ }^{2} \mathrm{La}$ estructura urbana refiere la manera en que se distribuyen y articulan los distintos componentes de una ciudad: la función, la forma y los flujos que, además, están expuestos a mutaciones e incrementos a lo largo del tiempo, según sea su adaptación al contexto socioeconómico del momento (Bourne, 1982). La estructura urbana refleja la evolución histórica de una sociedad específica, el origen de su asentamiento, las costumbres que la caracterizan y, por ende, el modo en que se ha adjudicado el territorio y las relaciones existentes entre las actividades y los componentes de la ciudad (Bourne, 1982 y Kunz, 1995). 
Gallegos Jiménez

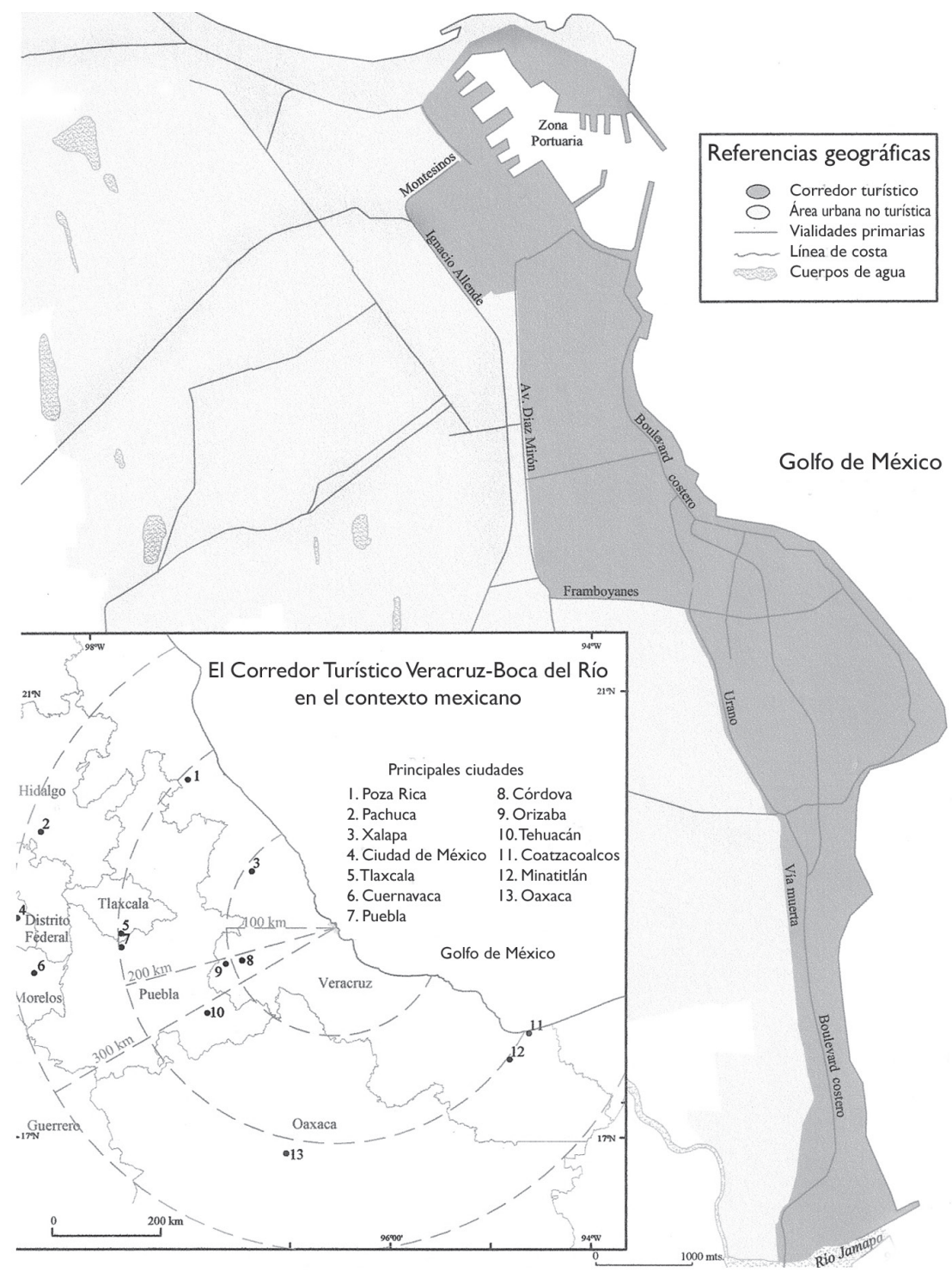

Fuente: Elaborado con base en el Programa de Ordenamiento de la Zona Conurbada de los Municipios de Veracruz-Boca del Río-Medellín-Alvarado e investigación directa, 2005.

Figura I. Localización del corredor turístico Veracruz-Boca del Río 
construcción de la zona portuaria y la estación y red férreas lo que indujo un crecimiento en dirección sur, mientras que para Boca del Río la presencia del río Jamapa y del estero ubicado en su rivera sur propició la expansión del poblado hacia el norte.

La ciudad deVeracruz fue fundada en 1519 por Hernán Cortés; no obstante, fue hasta 1597, después de haber sufrido dos cambios de sede, cuando se asentó y consolidó en su actual ubicación (Blázquez y Díaz, I 996 y Blázquez, 2000). Aunque durante el siglo XVII y la mayor parte del XVIII la localidad mantuvo una abundante movilidad de personas, su población y superficie prácticamente no se incrementaron, pues la mayoría de quienes arribaban a ella la abandonaban muy pronto por considerarla insalubre y de clima inhóspito (Blázquez y Díaz, 1996 y Blázquez, 2000).

Si bien desde finales del siglo XVIII e inicios del XIX la estructura de la ciudad mejoró con el empedrado de sus calles principales, la construcción de nuevos edificios y el diseño de paseos peatonales, fue a partir de la segunda mitad del siglo XIX cuando Veracruz registró avances significativos en su fisonomía con obras como la construcción del ferrocarril México-Veracruz, la instalación del telégrafo, el tranvía urbano de tracción animal, la caída de la muralla y, sobre todo, la modernización portuaria emprendida en 1895; baste decir que con este trabajo se le ganaron al mar cerca de 100 hectáreas (Blázquez y Díaz, 1996 y Blázquez, 2000).

El primer tercio del siglo $x x$ trajo consigo cambios notables en la estructura urbana deVeracruz, en particular, en la relacionada con las actividades turísticas. En este período, en la zona centro de la localidad se introdujo alumbrado, tranvía eléctrico y alcantarillado; además, se incorporaron nuevas colonias en su periferia al convertirse en un receptor de inmigrantes nacionales y extranjeros.Así, las notables mejoras en sus problemas de insalubridad, equipamiento urbano y el aumento de la población en tránsito intercontinental con horas de ocio produjeron las primeras manifestaciones turísticas; no sólo se abrieron nuevos establecimientos de alimentación y hospedaje, sino también algunos recreativos como bares, cafés, paseos peatonales y los balnearios Nereidas, Regatas y Villa del Mar (García, 1996). Pese a que en esta época prevaleció la función portuaria de la localidad, otras actividades económicas, como el turismo, comenzaron a delimitar la organización del espacio. 
El entorno económico generado a raíz de la Segunda Guerra Mundial, que fortaleció la incipiente industria del país y, con ello, la migración rural-urbana (que llegó a su umbral máximo a finales de los ochenta), significó para Veracruz desde los años sesenta, el inicio de su proceso de metropolización (Bamford, 2002 y Sobrino, 2003). Las modificaciones en la estructura urbana de la ciudad se basaron en una sucesiva renovación portuaria, en la construcción de parques industriales y en un acelerado crecimiento demográfico. Contrario al desarrollo general de la estructura urbana, la relacionada en particular con el turismo comenzó una larga etapa de estancamiento y rezago en el contexto nacional.

La ciudad deVeracruz había mantenido la fama de ser el balneario costero tradicional "más antiguo de México"; sin embargo, durante los años setenta y ochenta sus evidentes carencias respecto de los Centros Integralmente Planea$\operatorname{dos}^{3}$ la convirtieron en una localidad con poca difusión turística internacional que se caracterizó por servir a un "turismo popular" nacional, con una planta turística de baja categoría.

Desde mediados de los ochenta, la fase neoliberal del capitalismo ha producido cambios sustanciales en la administración pública y en la gestión del espacio. El crecimiento de la estructura observado en el AMVBR se ha cimentado en el desarrollo de complejos comerciales, corredores de cadenas de tiendas de autoservicio o productos especializados, instalaciones académicas, centros de negocio, comercios y servicios turísticos sobre el bulevar costero y nuevas áreas habitacionales, tanto de pequeños fraccionamientos y condominios de clase media y alta, como de unidades habitacionales en la periferia urbana.

En lo turístico, este nuevo orden económico ha derivado en una reactivación del sector mediante una estrategia de apertura a la inversión privada para mejorar, ampliar y diversificar la oferta de atractivos y equipamiento de la ciudad. De esta forma, la estructura urbana de la ciudad relacionada con el turismo se ha transformado notablemente con acciones tales como la construcción del centro de exposiciones Expover (hoy World Trade Center, WTC); la ampliación del bulevar costero hasta la desembocadura del río Jamapa; la reestructuración y remode-

${ }^{3}$ Aquellos centros turísticos litorales desarrollados por el Estado a partir de los setenta, con el fin de captar divisas extranjeras a través de sitios costeros turísticos competitivos en el mercado internacional: Cancún e Ixtapa en una primer fase, y Loreto-Nopoló, Los Cabos y Huatulco en una segunda (López, 2002). 
lación del paseo del Malecón; y la edificación de un acuario, plazas comerciales, bares, discotecas y hoteles de franquicias nacionales e internacionales, así como condominios verticales asociados a un turismo de segunda residencia.

\section{EI espacio turístico del AMVBR}

Hasta ahora se ha hecho referencia al proceso histórico de la estructura urbana y del turismo del área metropolitana Veracruz-Boca del Río; pero es claro que hoy en día no toda la superficie de la ciudad está directamente involucrada con dicha actividad. En casos de sitios urbano-litorales como Veracruz, el espacio turístico suele adquirir formas alargadas dado su desarrollo paralelo a la costa, es decir, morfologías propias de corredores que en su interior concentran la mayor parte de la planta turística y que, por lo tanto, se distinguen claramente de las zonas que albergan a la población fija (Boullón, 1990 y Lozato-Giotart, 1990). Desde esta lógica, la forma del espacio turístico del AMVBR puede asumirse como la de un corredor turístico, puesto que ésta se proyecta en una franja alargada, paralela a la costa, que con dificultad será rebasada por el turista durante su estancia en la localidad, ya que en ella se concentran los atractivos turísticos y la mayor cantidad de comercios y servicios de esta índole.

De acuerdo con la disposición territorial de los principales ejes viales, la ubicación de los recursos turísticos, los diversos usos del suelo existentes en la localidad y las observaciones resultantes de una serie de recorridos de superficie, el corredor turístico Veracruz-Boca del Río ocupa un área aproximada de I 355 hectáreas (cerca de 19\% de la superficie del área urbana), que se extienden sobre una longitud de 16 kilómetros y un ancho entre los 100 metros y los tres kilómetros. En sus extremos norte y sur se encuentran los centros históricos deVeracruz y Boca del Río, respectivamente; en su costado este, el litoral del Golfo de México, y en el oeste, fungiendo como límite, las avenidas Ignacio Allende, Juan Soto, M.Abasolo, Salvador Díaz Mirón, Paseo Framboyanes, Urano,Vía Muerta y el Boulevard Miguel Alemán (Gallegos y López, 2006; figura I).

\section{Estructura interna del CTVBR}

Según Echarri y Salinas (2003), un modo atinado de estudiar la estructura interna de un espacio turístico es mediante la identificación de las unidades territoriales que lo 
conforman, ya que son éstas las que determinan en gran medida la dinámica y su crecimiento espacial. Asimismo los autores afirman que la configuración turística en un entorno urbano por lo general responde a la existencia de tres elementos básicos: a) focos o núcleos, aquellos sitios de centralidad turística que concentran los principales atractivos, la mayor afluencia de visitantes y el más alto consumo de bienes y servicios; b) recorridos conectores, vías que enlazan los diferentes núcleos y, en consecuencia, potencian el número de servicios y comercios turísticos; y c) zonas de influencia,áreas que se generan alrededor de los núcleos o en las inmediaciones de los recorridos conectores, $y$ en ellas se combina el uso habitacional con el de comercio y servicios.

A partir de tales consideraciones, el corredor turístico Veracruz-Boca del Río se encuentra estructurado por (Gallegos y López, 2006; figura 2):

I. Cinco núcleos turísticos: dos primarios (Centro Histórico Veracruzpuerto y Acuario-Villa del Mar) y tres secundarios (Central deAutobuses, Centro Comercial Las Américas-WTC y Centro Histórico Boca del Río). Sin duda, el núcleo Centro Histórico Veracruz-puerto es el de mayor importancia en todo el corredor, puesto que al ser el sector más antiguo de la ciudad, aglutina la mayor cantidad y variedad de atractivos, así como la más alta densidad de comercios y servicios afines al turismo.

2. Ocho recorridos conectores: dos de primera categoría (bulevar costero y Boulevard Adolfo Ruiz Cortines) y seis de segunda categoría (Xicoténcatl, General Prim-Flores Magón, Díaz Mirón, Simón Bolívar, Paseo Framboyanes y Costa Verde-Urano). Con alrededor de I 5 kilómetros de longitud, el bulevar costero es el de mayor peso en la estructuración del CTVBR, pues a través de él se conectan los diversos núcleos (con excepción del de la Central de Autobuses).

3. Dos zonas de influencia: una de primer orden derivada de la proximidad con el bulevar costero, y otra de segundo orden, asociada con áreas más distantes de la costa, ubicadas entre conectores de segunda categoría.

Por último, es importante mencionar que dentro del CTVBR hay porciones donde el uso del suelo es totalmente habitacional y la planta turística inexistente; aunque por su ubicación intermedia es conveniente considerarlas parte del corredor pues participan de manera indirecta en su dinámica. 
Organización espacial del corredor

turístico Veracruz-Boca del Río

\section{Recursos turísticos y equipamiento del CTVBR}

En el interior del CTVBR existen diversos atractivos turísticos naturales y culturales, así como una amplia gama de servicios y comercios que dan sustento a su dinámica (figura 2).A continuación se presenta una breve descripción de los principales recursos y del equipamiento urbano vinculado al turismo.

\section{Atractivos naturales}

Playas y mar.Históricamente, las playas de la ciudad han constituido un atractivo esencial en la promoción turística de la localidad.Si bien la costa del Golfo de México en general presenta condiciones físicas favorables para su uso como balneario (pendientes suaves, espacios anchos y granulometría fina), lo cierto es que los rasgos originales de la mayoría de las playas del CTVBR han sido modificados a tal grado, que en distintos sectores de la ciudad éstas han desaparecido o han quedado reducidas a una franja no mayor a 30 metros.

Río Jamada. Se localiza en el extremo sur del CTVBR y aunque no se le explota directamente como balneario su caudal tranquilo se presta para la renta de motos acuáticas; en su rivera norte existe un pequeño andador desde el cual se puede observar el paisaje de su desembocadura o su curso interno, vista que han aprovechado una serie de restaurantes ahí emplazados.

Atractivos culturales

Arquitectura histórica y museos. A pesar de que Veracruz fue la primera ciudad colonial fundada en México, son pocas las construcciones antiguas que aún permanecen. ${ }^{4}$ El CTVBR conserva algunos edificios históricos que en su mayoría han sido habilitados para albergar museos, oficinas de los ayuntamientos y comercios. Dentro de los que acogen museos se encuentran el Baluarte de Santiago (principal vestigio de la muralla veracruzana), el Fuerte de San Juan de Ulúa (fortificación militar más antigua de la zona), el Faro Venustiano Carranza (Museo de la

${ }^{4}$ Esto se debe a la numerosa pérdida de inmuebles ocurrida en los diversos ataques bélicos; al deterioro de los edificios, producto de las condiciones climáticas; $y$ a que no se construyeron grandes mansiones, pues la deficiente higiene y el inhóspito clima motivó que del siglo XVI al XIX las personas fijaran sus residencias en ciudades cercanas como Xalapa o Córdoba. 
Gallegos Jiménez

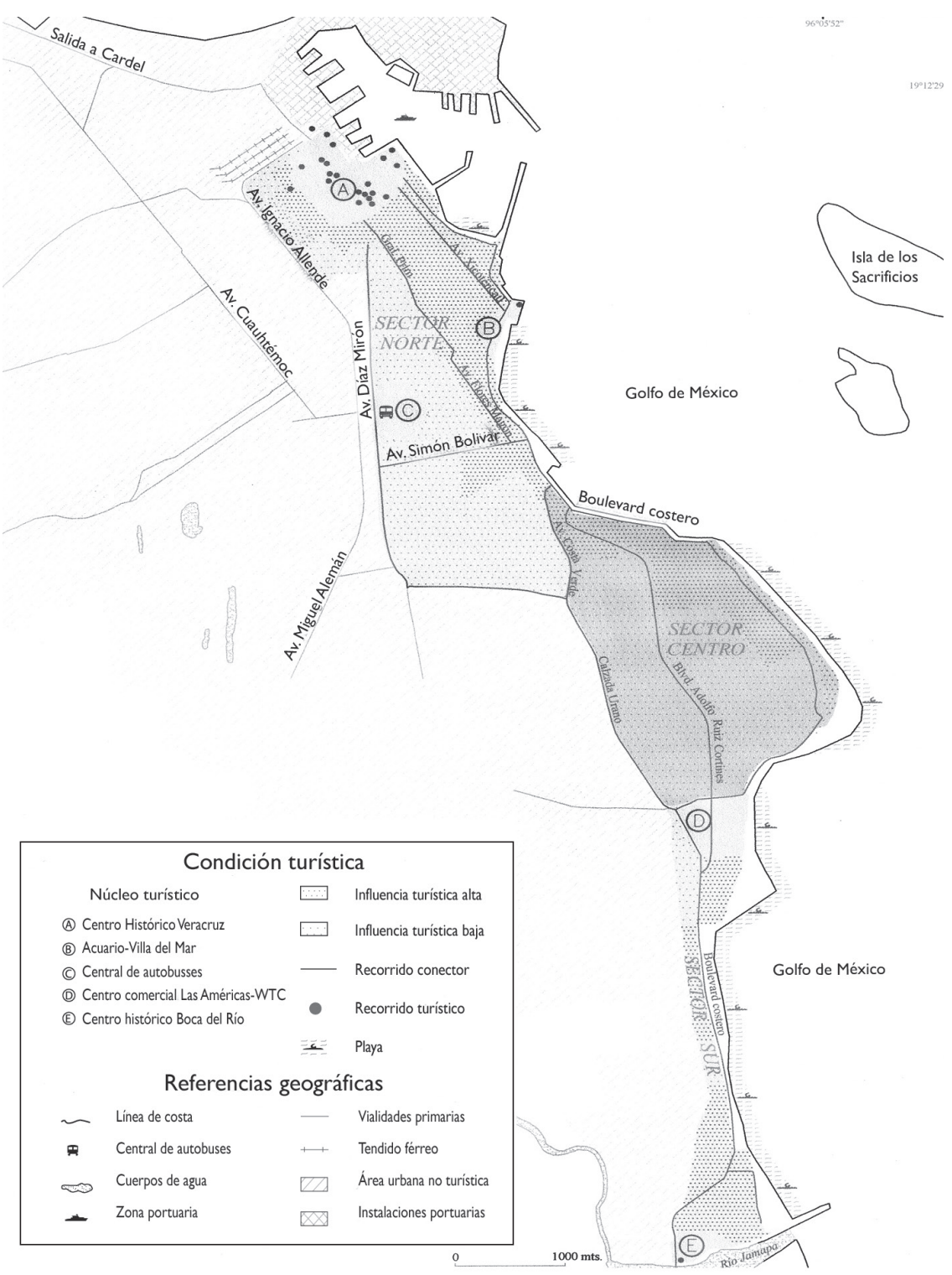

Fuente: Elaborado con base en investigación directa, 2005

Figura 2. Organización espacial del Corredor Turístico Veracruz-Boca del Río, 2005 
Revolución y de la Tercera Zona Naval), el de la Ciudad "Coronel Manuel Gutiérrez Zamora”, el de Pemex, el Histórico Naval de Veracruz y el Espacio Cultural Recinto de La Reforma. En cuanto a los edificios antiguos ocupados por como oficinas públicas o comercios, destacan los palacios municipales de Veracruz y Boca del Río, el edificio de Correos y Telégrafos, la estación del ferrocarril, el Portal de Miranda y Los Portales.

Áreas peatonales. Se trata de caminos y sitios públicos muy concurridos por los turistas, y pueden clasificarse con base en dos aspectos: los asociados con la costa y los que mantienen cierta conexión con plazas públicas. En relación con los primeros, sobresalen el Paseo Insurgentes Veracruzanos y el Malecón, ambos de cara a las instalaciones portuarias, muy tradicionales y donde se venden recuerdos y artesanías de la ciudad; el Paseo Hornos-Villa del Mar, integrado a la dinámica turística del Acuario de Veracruz; y, finalmente, el paseo o bulevar costero. Con referencia a las plazas, son importantes la de Armas (zócalo), los portales veracruzanos, la de la República y el parque Zamora.

Carnaval de Veracruz. El carnaval es la festividad más célebre no sólo de la ciudad sino del estado. Durante nueve días, por lo general de febrero, una buena porción del bulevar costero se acondiciona con gradas para disfrutar el desfile de carros alegóricos y comparsas participantes. Originalmente se trató de una fiesta popular local, pero en la actualidad es un acto masivo-comercial que, sin duda, tiene gran importancia para la ciudad, pues los ingresos que se derivan de él son muy elevados.

\section{Actividades turísticas}

En el CTVBR existen varios servicios públicos o de la iniciativa privada asociados a los principales atractivos; los más destacados son los paseos turísticos, ya sean vía terrestre, mediante autobuses panorámicos, o acuáticos, en lanchas o embarcaciones de pequeña escala. Por otra parte, a lo largo del año se organizan actividades culturales en las diferentes plazas públicas para que los turistas y la población local asistan a bailar danzón o a presenciar bailes folclóricos.

Equipamiento urbano vinculado al turismo. Oferta de hospedaje. De 1992 a 2005 las habitaciones disponibles en hoteles de cuatro y cinco estrellas registraron un 
notable incremento; ${ }^{5}$ hoy en día comprenden 21 y $25 \%$ respectivamente. ${ }^{6}$ A partir de 1990, el arribo de cadenas hoteleras nacionales y extranjeras ha sido constante: en los últimos 15 años, se han construido 16 hoteles que pertenecen a algún grupo hotelero; de esta forma, la oferta de hospedaje de más alta categoría de la ciudad ha dado un vuelco significativo, pues de estar básicamente en manos de inversionistas locales, en un ámbito familiar, ha pasado a las de reconocidas franquicias.

Restaurantes, discotecas, cafés y bares. Según datos proporcionados por la Oficina de Visitantes y Convenciones del Estado de Veracruz, en el 2005 se registraron 97 establecimientos de alimentos y bebidas con categoría turística en el AMVBR, la gran mayoría situados dentro del CTVBR.Además de éstos, existe otra gran cantidad que es frecuentada por los turistas, sobre todo los ubicados en los centros históricos de Veracruz y Boca del Río. Las discotecas visitadas por turistas, que son las más caras y las de moda, se localizan sobre los recorridos conectores bulevar costero y Boulevard Adolfo Ruiz Cortines; en conjunto, suman más de I5. En relación con los cafés y bares, también hay una amplia diversidad; los más conocidos son El Gran Café de la Parroquia, La Parroquia de Veracruz, El Gran Café de los Portales, Andrade, Silvia, La Perla, Bar Azúcar, El Ancla, Corintos y Playa Paraíso.

Plazas comerciales y equipamiento para actos masivos. En los últimos 20 años, una estrategia comercial exitosa en las áreas urbanas han sido las plazas o centros comerciales. Dentro del CTVBR se ubican cuatro espacios de esta índole: el Centro Comercial Las Américas que desde su construcción, a principios de los años noventa, se mantiene como el más importante de la ciudad; la Plaza Acuario, cuya dinámica gira en torno al Acuario de Veracruz y al Museo de Cera; y las plazas comerciales Mocambo y Boca del Río.

Por otro lado, el turismo de negocios, que si bien en su origen no representan actividades propiamente turísticas, genera la llegada de personas con un tipo de consumo similar al del turista típico. En el corredor existen diversos inmuebles para convenciones, congresos y ferias, como elWorld Trade Center,

${ }^{5}$ En 1992, el número de habitaciones disponibles en hoteles de cinco estrellas era de 508 , y de 727 en los de cuatro estrellas; para el 2005, las cifras ascendieron a I 929 y I 652, respectivamente (Ayuntamiento de Veracruz, 1985 y http://www.sectur.gob.mx).

${ }^{6}$ Véanse Ayuntamiento de Veracruz (1985) y http://www.sectur.gob.mx 
Organización espacial del corredor

turístico Veracruz-Boca del Río

Veracruz (antes Expover), el Atarantas, Estación Ferroviaria, la Fortaleza de San Juan de Ulúa y el Museo Sala Melchor Ocampo del Registro Civil.

\section{Dinámica espacial del CTVBR}

De acuerdo con la ubicación de los recursos turísticos y de la estructura interna del corredor, se puede concluir que su dinámica espacial se organiza en función de tres sectores: el norte, el centro y el sur (figura 2).

\section{a) Sector norte}

Al ser el área más antigua de la ciudad, su composición interna combina de manera equilibrada el uso de suelo comercial y de servicios con el habitacional, particularmente en el centro histórico, donde se concentra una amplia gama de funciones urbanas: administrativa, comercial, turística, habitacional y portuaria; en general, el patrón de densidad habitacional del sector se incrementa conforme aumenta la distancia existente respecto al centro histórico y al bulevar costero.

Su equipamiento urbano, aunque antiguo en su mayoría, se encuentra en buenas condiciones; la traza urbana consiste en manzanas pequeñas y calles angostas que al exterior del centro histórico son cortadas por amplias avenidas de orientación norte-sur; esta configuración evidencia que las etapas de crecimiento del sector se han dado conforme a la dirección de estas vialidades. Cabe destacar que su espacio costero ha sido modificado de manera radical y que la saturación territorial del sector está al máximo.

Sin duda, constituye la porción más tradicional y visitada del CTVBR; la de mayor interés para todo tipo de visitante. Su dinámica turística marcada por el entorno arquitectónico-cultural del centro histórico de Veracruz, la contemplación del tráfico portuario y actividades recreativas propias de la costa.Así, a partir de la Plaza de Armas, del Paseo del Malecón, del Paseo Acuario-Villa del Mar, del bulevar costero y de sus playas, la influencia del turismo se extiende en la mayor parte de las manzanas; de esta forma, aglutina la mayor cantidad y variedad de atractivos, así como de comercios y servicios turísticos.

Pese a que su planta turística es basta y diversa, ésta corresponde a la más antigua de la ciudad; $y$ aunque algunos hoteles y restaurantes han remodelado sus instalaciones, aún conservan su fisonomía arquitectónica original. Por tal motivo, aunque es posible localizar equipamiento turístico de la más alta categoría, lo cierto es que la mayor parte es utilizado por turistas de nivel socioeconómico medio-bajo. El ejemplo más representativo de este turismo popular se presenta 
en la playa Villa del Mar, cuyos visitantes arriban en su mayoría en autobuses de paquetes turísticos que ofertan traslado y hospedaje en esta zona del corredor.

b) Sector centro

Es el sector más reciente del CTVBR, y puede ser catalogado como un subcentro urbano de carácter comercial y de servicios de toda el AMVBR; por esta razón, en su composición domina el uso del suelo comercial y de servicios $y$, en menor medida, el habitacional. En su interior se encuentra el recinto de convenciones más famoso de la ciudad (WTC), la zona habitacional más lujosa del área metropolitana, un importante segmento escolar, las principales tiendas departamentales y una densa presencia de comercios y servicios sobre los recorridos conectores. Es el único sector del corredor que aún tiene amplios lotes baldíos, los cuales están sujetos a una fuerte especulación de costo y uso por la tendencia turística y comercial de la zona.

Salvo los recorridos conectores Costa Verde-Urano, Boulevard Adolfo Ruiz y el bulevar costero, que atraviesan el sector en dirección norte-sur, el resto de las vialidades son cortas en forma de retornos o cerradas con cierto orden ortogonal. El equipamiento urbano se encuentra en excelentes condiciones, lo que le confiere un eficiente y moderno orden urbano que deja ver las construcciones de mayor dimensión del CTVBR, como el Centro Comercial Las Américas-WTC, las plazas comerciales Costa Verde y Boca del Río, el estadio de futbol, el lienzo charro y la expo ganadera.

En el estricto sentido de lo turístico, este sector no depende de los visitantes; empero, sus características de organización interna generan una estrecha relación con esta actividad, por lo tanto, después del centro histórico Veracruz, esta porción del CTVBR presenta la más alta densidad de servicios y comercios afines al turismo. En su lado costero se ubican los hoteles, restaurantes, bares y discotecas de mayor categoría y moda del área urbana, así como los condominios turísticos más lujosos, concluidos o en construcción. El principal atractivo turístico del sector son sus playas, las cuales al ser las más anchas y preservadas del corredor han dado paso al establecimiento de nuevas cadenas hoteleras.

Por las particularidades citadas, la dinámica turística del sector está asociada por completo con un turismo de alto nivel socioeconómico o de negocios. A diferencia del sector norte, la mayoría de los visitantes que arriban a esta zona lo hacen en un ámbito recreativo independiente, ajeno a algún paquete turístico, o bien porque su estancia responde a algún evento no necesariamente 
Organización espacial del corredor

turístico Veracruz-Boca del Río

turístico (congreso y expo). Si bien en la actualidad sus playas son de acceso libre, se prevé que, al agotarse los segmentos baldíos, su paso y uso quedará sujeto a los hoteles y condominios del área.

\section{c) Sector sur}

Se extiende desde la inmediación sur del WTC hasta la desembocadura del río Jamapa, en cuyo margen izquierdo se localiza el centro histórico de Boca del Río. En estricto sentido, representa una ruptura del continuo habitacionalcomercial-turístico del tipo medio-alto del bulevar costero, pues el uso del suelo predominante es el habitacional. Éste disminuye y se combina con el de comercio local y de servicios administrativos (correspondientes al ayuntamiento), conforme se reduce la distancia respecto a dicho sitio histórico. Si bien este último fungió durante muchos años como el principal centro urbano de Boca del Río, en la actualidad sólo podría considerársele un subcentro urbano dentro del área metropolitana.

Su traza urbana es totalmente irregular pues es resultado del escaso espacio existente entre el antiguo camino hacia Boca del Río y la línea costera, franja sobre la cual se dio el crecimiento primario de esta porción de la ciudad. Así, es el sector territorial que de manera más evidente muestra la discontinuidad entre la estructura urbana del CTVBR y el resto del área metropolitana, ya que el panorama de edificios nuevos y diseños modernos se rompe de forma abrupta con un paisaje de casas viejas y maltratadas. Por su parte, el extremo sur del sector, muestra una configuración propia de una pequeña localidad que, por mucho tiempo, fue independiente del área urbana de la ciudad de Veracruz.

Su soporte turístico está dado por un reducido número de lujosos condominios de alrededor de 100 metros cuadrados que ofertan su vista al mar, a una serie de restaurantes emplazados en la desembocadura del río Jamapa, al edificio y la plaza del ayuntamiento, $y$ a una nueva avenida costera que ha abierto al uso recreativo la única porción de playa apta como balneario. En general, el perfil socioeconómico de los visitantes es medio-bajo, y su afluencia y estancia promedio están muy por debajo de las registradas en los otros sectores del corredor turístico.

\section{Conclusiones}

En el contexto turístico nacional, el corredor turístico Veracruz-Boca del Río tiene una posición destacada, puesto que en su espacio confluyen diferentes 
tipos de turismo, con connotaciones territoriales diferentes:sol y playa, históricocultural, urbano-portuario y de negocios. Sin embargo, la captación turística del área metropolitana Veracruz-Boca del Río se encuentra muy por debajo de otros centros litorales del país como Cancún, Acapulco y Huatulco.

Con todo, se reconoce que en el corredor mencionado, con la incorporación de nuevos equipamientos urbanos, así como de distintos comercios y servicios afines al turismo de negocios, se prevé un significativo y constante aumento en el arribo de visitantes.

Bajo una creciente participación de la iniciativa privada en los últimos I5 años, el espacio turístico del área metropolitana Veracruz-Boca del Río ha experimentado una significativa diversificación de su oferta y una notable transformación territorial, que le han permitido integrar a su dinámica, además de un turismo popular nacional, segmentos sociales con niveles de ingresos medio y alto, vinculados a prácticas especializadas o de negocios.

Si bien la expansión territorial de la actividad turística sobre la línea costera ha incorporado nuevos solares, es importante resaltar que su ocupación ha quedado en manos de especuladores inmobiliarios o de inversionistas turísticos asociados con franquicias nacionales e internacionales de hospedaje y alimentación, que, paulatinamente, han acentuado la fragmentación del espacio y la constitución de segmentos privilegiados en equipamiento urbano, sobre todo dirigidos a personas con un alto poder adquisitivo.

Aunque la figura del corredor turístico Veracruz-Boca del Río aduce a un continuo territorial que por su estructura y dinamismo urbano puede distinguirse del resto del área metropolitana, en su interior coexisten diferencias que ponen al descubierto tres distintos sectores turísticos: el norte se asocia con la imagen clásica y popular de Veracruz-puerto, accesible a cualquier estrato socioeconómico y concentrador de los principales atractivos; el centro, vinculado a la imagen nueva de la ciudad, a un turismo de sol y playa, urbano y de negocios selectivo, accesible a los estratos sociales medio y alto; finalmente, el sur, propio de un sector recién incorporado a la dinámica turística de la localidad, con pocos atractivos, captador de desarrollos inmobiliarios de condominios turísticos.

Por último, en consideración con el ritmo de ocupación del espacio disponible y la dirección del crecimiento de la franja turística, es posible suponer que en un futuro mediato el corredor turístico iniciará un proceso de expansión en dirección sur hacia la localidad de Antón Lizardo. 


\section{FUENTES CONSULTADAS}

Ayuntamiento de Veracruz (1985). Plan de Desarrollo Urbano del Centro de Población de Veracruz, Veracruz.Veracruz:Ayuntamiento de Veracruz.

Bamford, H. (2002). La historia de México. México: Diana.

Blázquez, Carmen (2000). Breve historia de Veracruz. México: Fondo de Cultura Económica/El Colegio de México (serie Breves Historias de los Estados de la República Mexicana).

— y Concepción Díaz (1996). “La ciudad y puerto de Veracruz: una retrospectiva”. Veracruz. Primer puerto del continente. México: Espejo de Obsidiana/ICA/Fundación Miguel Alemán, 67-I26.

Boullón, R. (1990). Planificación del espacio turístico. México:Trillas.

Bourne, L. ( I 982). Internal structure of the city. Nueva York: Oxford University Press.

Echarri, Maite y Eduardo Salinas (2003). "Turismo de patrimonio en el Centro Histórico de La Habana”, en Alberto Capacci (coord.). Paisaje, ordenamiento territorial y turismo sostenible. Génova: Brigati, 69-80.

Gallegos, O. y A. López (2006). "Fragmentación y segregación en el espacio litoral-urbano: el caso del Corredor TurísticoVeracruz-Boca del Río”. Estudios Multidisciplinarios en Turismo, I, I39-I 74.

García, B. (1996). “Dinámica y porvenir del Puerto de Veracruz: crecimiento $y$ transformaciones en el siglo xx". Veracruz. Primer puerto del continente. México: Espejo de Obsidiana/ıcA/Fundación Miguel Alemán, 223-250.

Kunz, I. (1995). Economía urbana y mercados inmobiliarios. México: Instituto Tecnológico de Estudios Superiores.

López, Álvaro (2002). “Análisis de los flujos turísticos en el corredor Los Cabos, Baja California Sur”. Investigaciones Geográficas, México, Instituto de Geografía, UNAM, abril, 47, I3 I- I49.

Lozato-Giotart, J. (1990). Geografia del turismo. Barcelona: Masson.

Secretaría de Desarrollo Urbano de Veracruz (s/f). Programa de Ordenamiento de la Zona Conurbada de los Municipios deVeracruz-Boca del Río-MedellínAlvarado. México: Gobierno del Estado de Veracruz-Llave.

Sobrino, J. (2003). Competitividad de las ciudades en México. México: El Colegio de México. 